\title{
GLAD!
}

Revue sur le langage, le genre, les sexualités

02 | 2017

Varia

\section{Les Transparences}

Transparencies

\section{Lorraine Alexandre}

\section{(e) OpenEdition}

Journals

Édition électronique

URL : http://journals.openedition.org/glad/559

DOI : $10.4000 /$ glad.559

ISSN : 2551-0819

\section{Éditeur}

Association GSL

\section{Référence électronique}

Lorraine Alexandre, « Les Transparences », GLAD! [En ligne], 02 | 2017, mis en ligne le 01 juin 2017 consulté le 21 janvier 2021. URL : http://journals.openedition.org/glad/559 ; DOI : https://doi.org/ 10.4000/glad.559

Ce document a été généré automatiquement le 21 janvier 2021.

\section{(c) (i) (9)}

La revue GLAD! est mise à disposition selon les termes de la Licence Creative Commons Attribution Pas d'Utilisation Commerciale - Pas de Modification 4.0 International. 


\section{Les Transparences}

Transparencies

\section{Lorraine Alexandre}

Il y avait, dans un de mes Bibi Fricotin, une invention fabuleuse qui me faisait rêver, et qui me faisait très peur en même temps si j'imaginais

qu'on pouvait les retourner contre moi : les lunettes à lire la pensée. Depuis, j'ai trouvé dans des réclames plus ou moins salaces l'existence de

lunettes qui transpercent les vêtements, qui déshabillent. Et j'ai imaginé que la photographie pouvait conjuguer ces deux pouvoirs... ${ }^{1}$ 


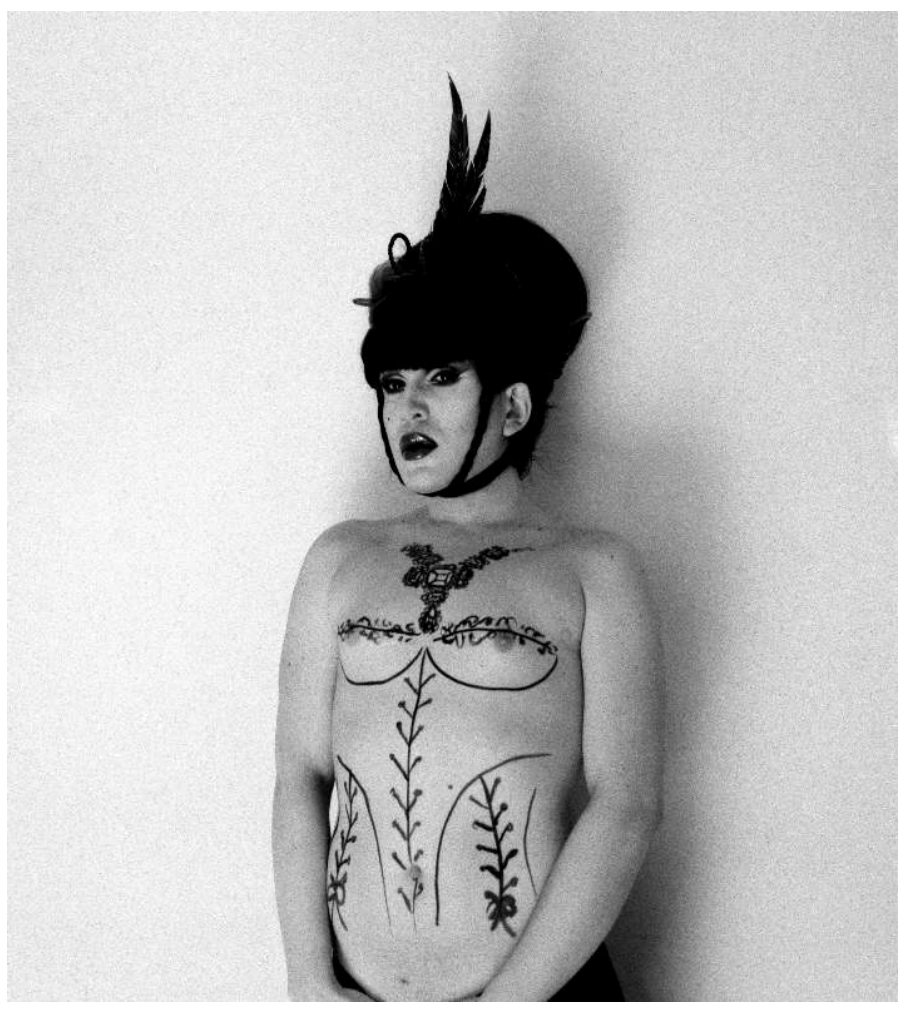

LES TRANSPARENCES \#1, MOdÈLE : AAKASHA.

Lorraine Alexandre, 2007, dessin sur la peau et photographie performative, noir et blanc, argentique, format et encadrement variables.

1 Ce fantasme exprimé par Hervé Guibert est aussi banal que profond. Il est révélateur de la nature curieuse de l'Homme cherchant à comprendre et à connaître les personnes qu'il rencontre ; il est révélateur surtout de l'impuissance à y parvenir... De là naissent les rêves et leurs solutions magiques, les clés faciles d'usage et répondant, de fait, à une logique voyeuriste.

En tant que plasticienne portraitiste, j'assume l'impossibilité à maitriser la complexité d'une personne et j'en use en mettant en scène cette impossibilité même. Mes créations jouent avec le voyeurisme du spectateur pour mieux le frustrer et le renvoyer à luimême en imposant une distance. De fait, mes portraits ne représentent pas des individus dans leur quotidien, ils représentent des états performatifs, des mises en scène du corps. Les modèles s'y dévoilent à travers un discours visuel, artistique. Presque toutes mes créations se jouent ainsi de l'imaginaire. Elles utilisent la puissance fictionnelle des masques pour révéler des identités. Michel Serres nous offre une métaphore plus subtile et pudique faisant écho au fantasme évoqué par Guibert lorsqu'il précise que « dévoiler ne consiste point à ôter un obstacle, enlever un décor, écarter une couverture, sous lesquels gît la chose nue, mais à suivre patiemment, avec un respectueux doigté, la disposition délicate des voiles.... " Les costumes, les maquillages, les mises en scène et en représentations sont autant de discours sur le corps et l'identité.

Dans ma pratique artistique, de façon générale, j'utilise les masques, les postures stéréotypées du corps comme des discours visuels, un langage codé faisant de ce corps l'écran d'une mise en scène identitaire culturellement reconnaissable. 
4 Ma série de photographies performatives Les Transparences (2007) s'annonce plus proche de Guibert par la forme, mais elle respecte plus volontiers Serres par le fond en s'ouvrant à une certaine volonté parodique qui dénonce tout voyeurisme.

5 À l'origine, j'ai conçu cette série pour affirmer mon travail déjà accompli dans une dizaine de séries avec les Drag Queens. Je voulais ouvrir les yeux de certains spectateurs qui s'obstinaient à voir de «vraies femmes» (pour les citer) dans mes précédentes créations. Avec Les Transparences, le corps torse nu ne permet plus de doute quant à la nature biologique masculine parfaitement assumée des modèles dont le travestissement est une performance d'ordre identitaire, mais aussi artistique et scénique $^{3}$. L'idée initiale consiste donc à confronter la nature biologique des sexes à la nature performative des genres. J'interroge alors la valeur arbitraire des associations entre sexes et genres. J'observe les lois socio-culturelles qui en dessinent les règles et tendent, au contraire, à associer sexes et genres comme si les uns dépendaient des autres niant la différence entre nature et culture.

\section{La transparence des masques}

6 L'artiste suisse Urs Lüthi, qui compte parmi mes principales références, considère que «le corps n'existe qu'autant qu'il est modifié, maquillé, travesti, chargé d'une signification perturbatrice qui ouvre le dialogue ${ }^{4} »$.

7 Pour les Transparences, j'ai donc fait poser des Drag Queens portant perruque et maquillage, mais restées torse nu. Sur leur torse, je dessine très sommairement un corset et un collier de luxe. Elles affichent ainsi leur corps d'homme contrastant avec la revendication d'une ouverture des codes de genre elle-même exprimée par la métamorphose du visage, leur maquillage, symbole d'un droit à repenser les règles sociales de la virilité. L'un des éléments de la genèse de cette série repose sur une conversation avec l'un de mes modèles récurrents à l'époque, la Drag Queen Cherry Poppers. Elle avait exprimé son principal fantasme qui consistait à avoir une tête de femme sur un corps d'homme et en jouait souvent dans ses costumes au point de provoquer des conflits avec son metteur en scène. Je n'aurais pas osé, avant cette conversation, demander à des travestis de genre de retirer leurs seins postiches sachant à quel point ils y sont attachés. Notons que, sur une dizaine de modèles, seuls deux ont accepté de les retirer (malheureusement, Cherry avait déjà quitté Paris au moment des prises de vue).

8 Les Drag Queens avec lesquelles j'ai travaillé affrontent le regard normatif social et, sans prendre la peine de le subvertir, l'interrogent et appellent à la nuance. Leur usage des attributs de la féminité est d'autant plus intéressant que les Drag ne se réfèrent pas à la femme dans son aspect quotidien, mais aux figures féminines du Star System. Ces dernières ont pour particularité d'être des fictions, des constructions spectaculaires ; les Drag remettent ainsi en cause toute tentative de naturalisme.

9 Notons que tous les modèles de bijoux et de corsets sont librement inspirés d'exemples extraits de la haute couture, de tableaux de l'âge classique (XVII et XVIII ${ }^{e}$ siècles, où le corset était commun dans les portraits des femmes dont c'était alors l'habit quotidien) et des fiers inventaires déployant la grandeur des joyaux les plus chers au monde. J'utilise ainsi la célébrité et la valeur iconique que ces accessoires possèdent et qui les transforment en archétypes culturels identifiables par le public. 
10 J'ai choisi le corset, par exemple, plutôt que tout autre vêtement dit féminin, pour l'éloquence et l'ambiguïté de son histoire. Le corset est à l'origine un objet à vocation médicale devenu attribut féminin particulièrement contraignant et, paradoxalement, handicapant. Cet objet est donc porteur d'une certaine ironie à mon sens. C'est ainsi dans une dimension critique que ces attributs sont si sommairement et grossièrement esquissés sur des torses masculins libres, le souffle entier et débordant généreusement des limites imposées par les corsets dessinés. Le corps humain est ici traité en tant que surface, en tant que support et comme lieu de projection tel un écran vivant, lisible.

11 Depuis l'Antiquité, nous voyons tourner cette image qui m'interpelle de la société comprise comme un théâtre. Cette métaphore communément acceptée habite l'ensemble de ma création selon différentes modalités. Avec Les Transparences, je me joue volontiers de l'ironique remarque d'Oscar Wilde : «La plupart des hommes et des femmes sont contraints de jouer des rôles pour lesquels ils ne sont pas faits. [...] Le monde est un théâtre, mais la pièce est mal distribuée ${ }^{5}$.»

\section{Les créations affleurantes}

12 La peau est un organe privilégié de la perception et donc dans l'élaboration d'une image du corps. C'est pourquoi j'ai choisi de la mettre au cœur de différents travaux plastiques tous regroupés sous le terme affleurant qui regroupe mes séries qui mêlent dessin ou écriture sur la peau, photographie et performance. Il signale la volonté d'utiliser la peau comme support directement rattaché au corps dont les marques éphémères révèlent des revendications identitaires. L'apparence véhiculée sur un tel support devient l'affleurement d'un ressenti psychique, d'une idée... formant un miroir sans tain ouvert sur l'esprit.

13 L'ensemble de ma pratique plasticienne interroge les liens entre l'art et le vivant. Mes portraits, comme je le précisais en introduction, ne montrent jamais des individus, mais des corps en représentations, des corps performatifs qui, se projetant dans une mise en scène artistique, portent leur discours sur l'identité et les façons de la revendiquer. En dessinant sur le corps, «nous appréhendons la création imaginaire à son lieu le plus originaire, le plus névralgique et le plus secret - son épicentre ${ }^{6} »$.

14 Dans le rapport au corps comme support, les enjeux des Transparences consistent à déterritorialiser et reterritorialiser les genres en se confrontant à un point de départ socialement et culturellement établi qui tend à croire que l'identité est stable si elle correspond à des normes.

Le corps comme support participe activement à la forme finale du travail plastique. La pratique du dessin en tant que telle est, dans ce contexte, riche en ce que le dessin profite de la plasticité du corps humain. Celui-ci, en sa qualité d'être vivant, est réactif aux stimuli. Il est également mouvant, le simple fait de la respiration du modèle peut influencer un dessin fait sur le torse, sans oublier les fluctuations innombrables des textures de la peau, des rencontres parfois conflictuelles des poils du pinceau avec ceux du corps.

16 La peau est le lieu d'expression de l'individu qui y inscrit au quotidien les faits de son existence. Ce qui rappelle qu'elle n'est pas simple frontière entre un dedans et un dehors mais le lieu même par excellence où se narre l'histoire. La peau est un texte libéré du sens et dont le langage ne s'articule qu'à condition d'y inscrire un signe. « La 
peau retire au corps son statut d'objet au moment où elle n'est plus perçue comme l'enveloppe des formes. Telle une surface avec ses propres reliefs, elle transforme le corps-objet en corps-texte ${ }^{7}$.»

Les Transparences \#2

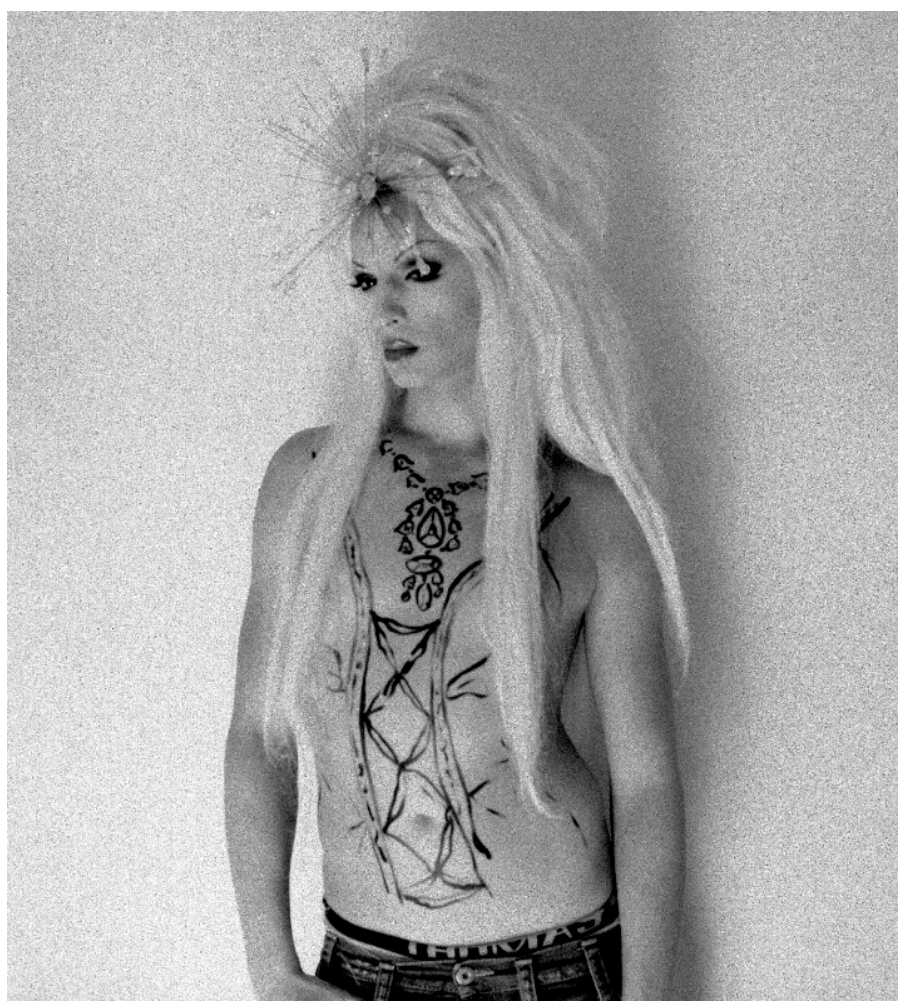

Les Transparences \#2, 2007, modèle : Poysen Irysh.

Lorraine Alexandre, 2007, dessin sur la peau et photographie performative, noir et blanc, argentique, format et encadrement variables. 
Les Transparences \#3

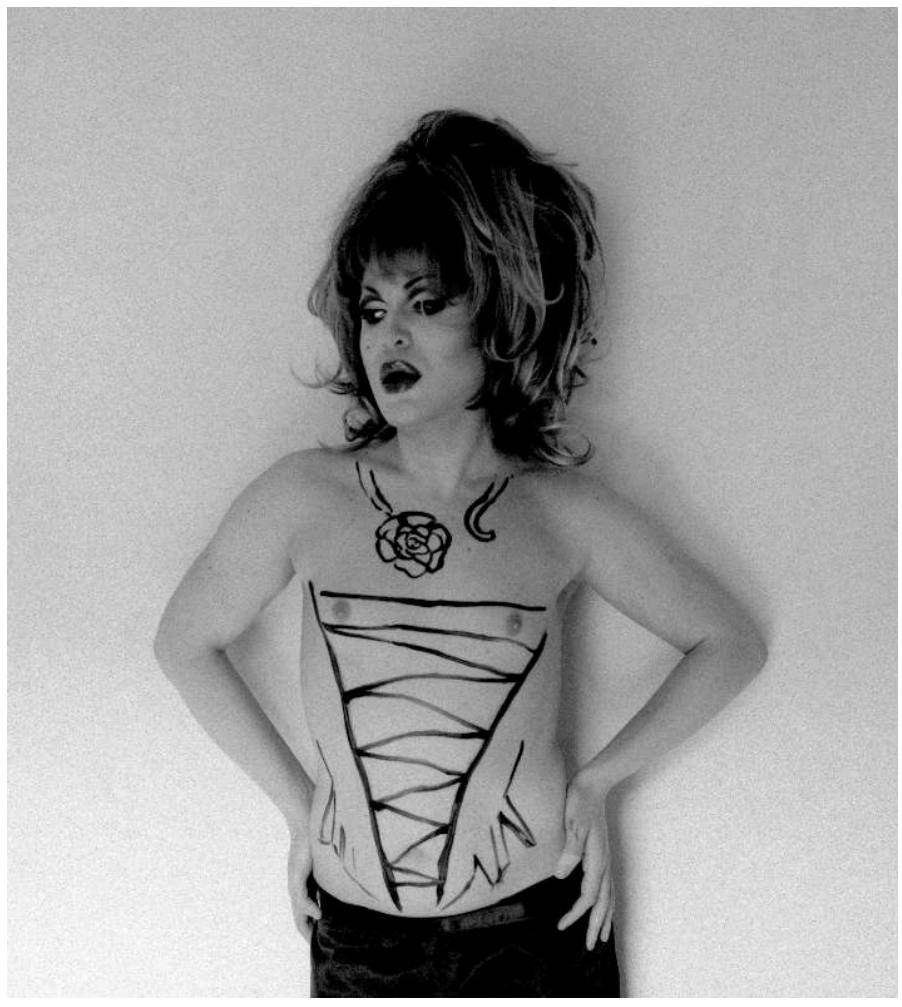

Les Transparences \#3, 2007, modèle : Aakasha.

Lorraine Alexandre, 2007, dessin sur la peau et photographie performative, noir et blanc, argentique, format et encadrement variables. 
Les Transparences \#4

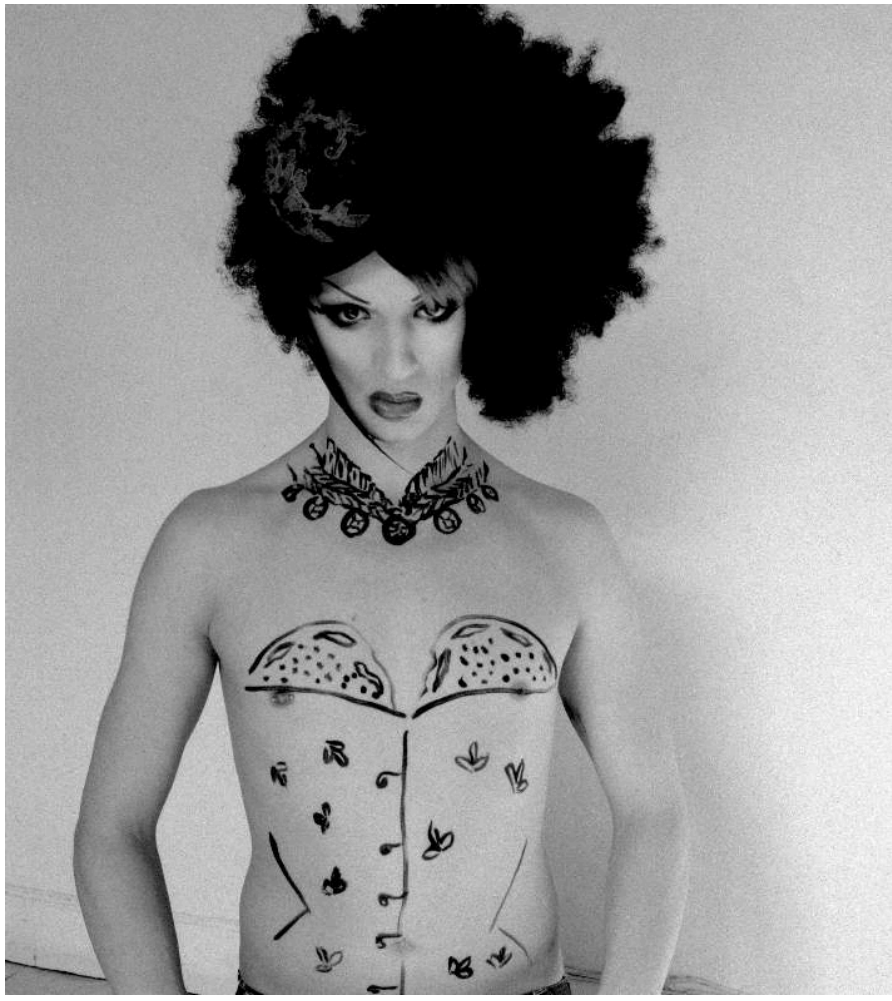

Les Transparences \#4, 2007, modèle : Poysen Irysh.

Lorraine Alexandre, 2007, dessin sur la peau et photographie performative, noir et blanc, argentique, format et encadrement variables. 


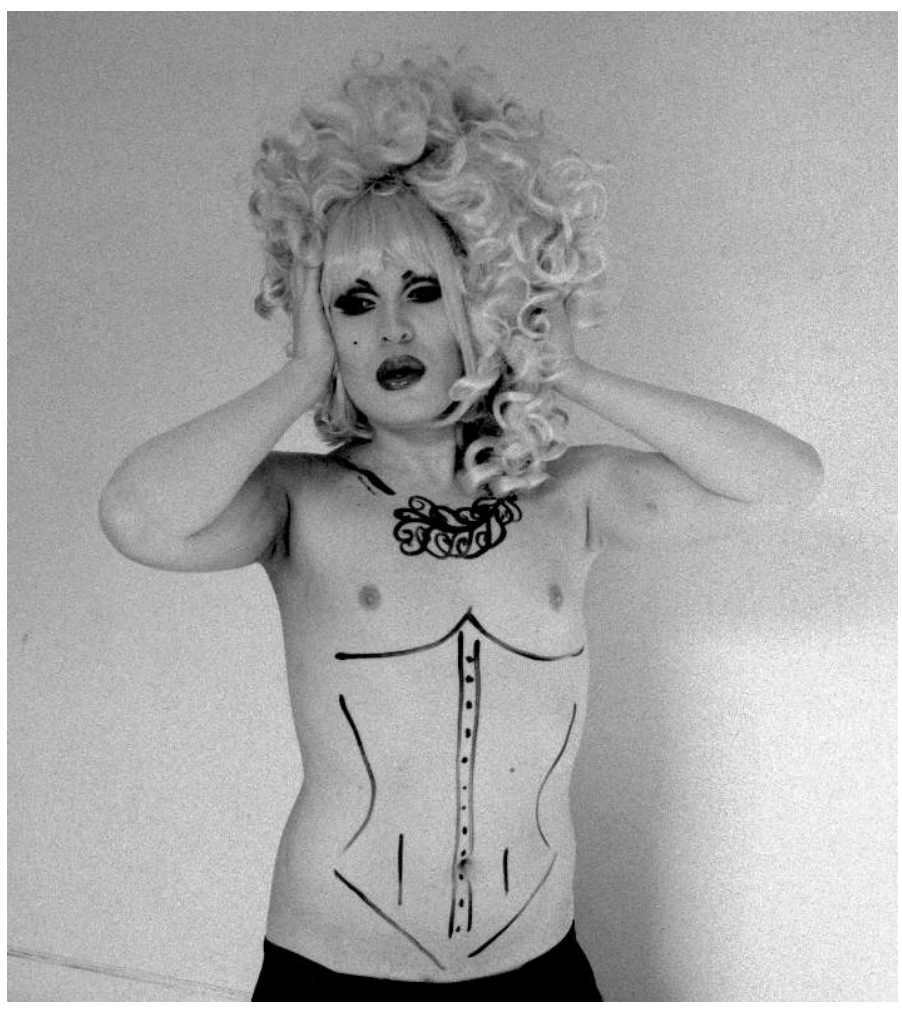

Les Transparences \#5, 2007, modèle : Aakasha.

Lorraine Alexandre, 2007, dessin sur la peau et photographie performative, noir et blanc, argentique, format et encadrement variables.

\section{Remarques techniques}

Notons certains aspects de la forme des Transparences ${ }^{8}$.

Le choix de représenter les vêtements des figures interprétées par des dessins sur la peau plutôt que d'avoir de vrais vêtements répond à la volonté de «transparence » du concept de ces séries, mais il permet également de penser la photographie plasticienne. La photographie s'est imposée avec le temps comme mon médium principal. Étant plasticienne de formation, je ne pratique pas les autres usages photographiques (reportage, mode, publicité, studio...), mais certaines autres techniques plastiques, à commencer par le dessin. Les amalgames fréquents du public entre les différentes formes de photographies m'ont amenée à m'interroger sur la nature du médium.

En tant que plasticienne, je me retrouve dans la logique citée par Soulages précisant qu'en art, « le problème n'est plus celui de l'objet transcendantal à photographier, mais celui de l'objet photographique à construire ${ }^{9} »$. Dans l'usage que j'en fais, la photographie développe une esthétique de la mise en scène en ce qu'elle est, plus qu'une reproduction de phénomènes, une image de concept. Cette remarque générale vaut pour tous mes travaux plastiques. Dans les cas précis des créations affleurantes, j'associe le geste graphique à ma mise en scène, ma performance photographique. Cette pluridisciplinarité cumulée dans une même pièce m'identifie comme plasticienne et me permet de me réapproprier les formes essentielles de ma propre genèse. 

après les prises de vues. «Ce que le croquis ou l'esquisse transmettent en premier lieu, c'est l'idée créative, surgissant du signe lui-même ${ }^{10}$. " La rapidité d'exécution du croquis et son refus de la maîtrise technique développent une énergie très différente de celle de la photographie venant ainsi l'enrichir et la compléter. Mes dessins sont de simples lignes sans profondeur. J'interpelle alors la visibilité des éléments choisis, la représentation et la faculté de représenter. L'esquisse est une forme synthétique de dessin qui suffit à l'identification de l'objet et qui instaure une esthétique du fragment.

21 Par ailleurs, un dessin trop détaillé pourrait masquer à l'excès le torse et compromettre l'ensemble du projet des Transparences qui doivent laisser visible le contraste entre sexe et genre. L'acte graphique que je joue pour mes mises en scène photographiques vient stratifier les séries formant l'un des voiles de la création. Cette création qui, elle-même, signale la stratification des modes d'apparaître du corps social, genré, politisé, culturellement identifiable.

Dans la citation qui amorce ce texte, Hervé Guibert dit qu'il prête à la photographie le double pouvoir de lire les pensées et de voir à travers les vêtements. Le médium se voit alors investi d'une mission : devenir, au propre comme au figuré, le révélateur de son sujet. Guibert s'amuse avec une métaphore volontairement provocatrice et poursuit un rêve photographique auquel je ne crois pas, mais qui me touche. Plus proche des voiles de Serres, c'est par les mises en scènes du corps que je cherche à toucher ses identifications les plus intimes. Ici, si le corps apparaît torse nu, c'est moins pour en montrer la « vérité nue » que l'enjeu et l'importance des attributs esquissés. De ce fait, «la photo est plus un produit qui interroge le visible qu'un objet qui le donne ${ }^{11}$ ». Et c'est ainsi que je la pratique : pour soumettre à la question des sujets que je mets en scène, que je tends à dévoiler par la texture même de leurs voiles dans un strip-tease conceptuel.

\section{Conclusion}

Mon travail photographique ne tend pas à révéler un réel, mais à le penser, le proposer comme objet d'expérimentation et d'interprétation. Ma création artistique met alors en avant les tensions entre la valeur esthétique de toute forme d'apparence, la valeur artistique de ma création et celle d'une création identitaire jouée par le corps lorsqu'il manipule les attributs, révélant ainsi leurs langages.

Mes travaux thématisent ainsi le circuit de valeur d'un corps/livre, un corps support et médium de l'art, lieu de mise en scène d'une culture dont la valeur même naît de son élaboration conceptuelle ainsi libérée, sans le dévaloriser ni le renier, du poids biologique, du destin génétique.

Lorsque j'ai commencé, en 2003, à user du corps comme support, j'étais fascinée par un film, The Pillow Book, de Peter Greenaway, non pas en tant que spectatrice mais bel et bien en tant que plasticienne, et j'aimerais conclure sur une réplique de son héroïne : "Je veux écrire le Corps comme un Livre - Un Livre comme un Corps ${ }^{12}$." 
Les Transparences \#6

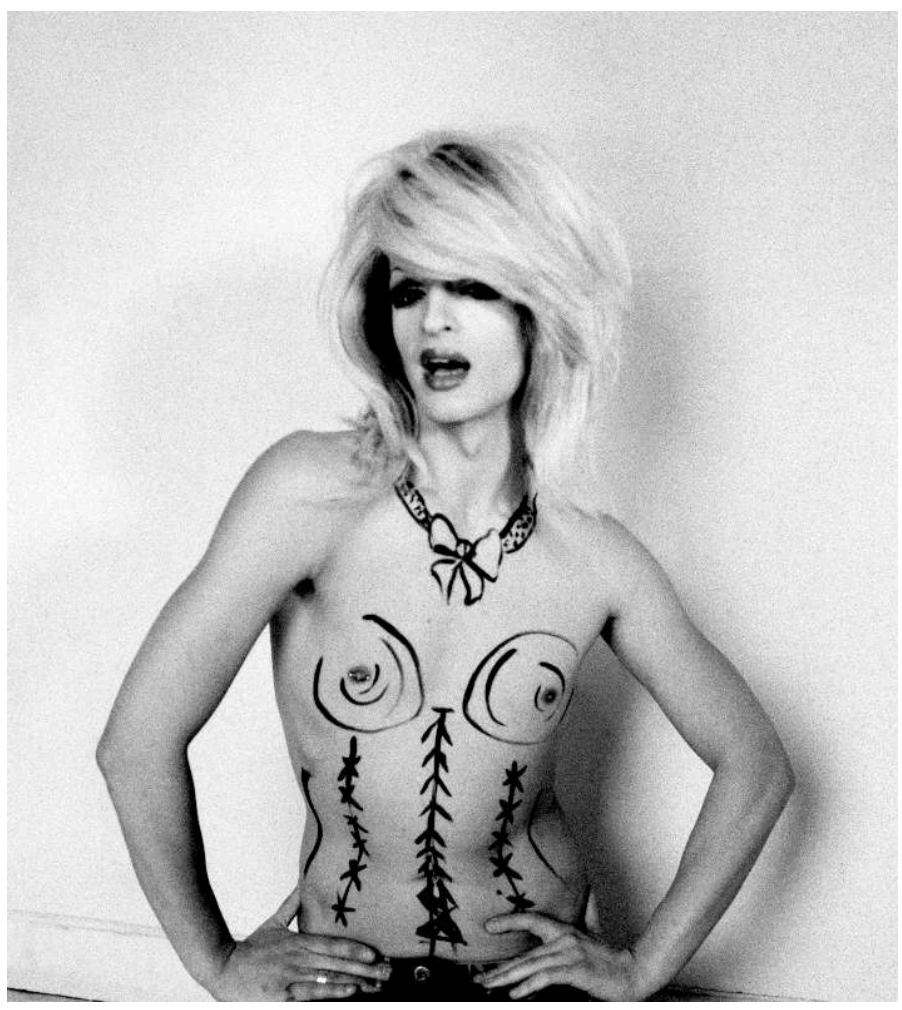

Les Transparences \#6, modèle : Poysen Irysh.

Lorraine Alexandre, 2007, dessin sur la peau et photographie performative, noir et blanc, argentique, format et encadrement variables.

\section{BIBLIOGRAPHIE}

ALEXANDRE, Lorraine. 2016. «L'Hommage à Marcel Mariën pour commencer... Traverser les genres » Plastir [en ligne] 42, consulté le 18 mai 2017. URL : http://www.plasticites-sciences-arts.org/ PLASTIR/Alexandre\%20P42.pdf

CIARAVINO, Joselita. 2004. Un Art paradoxal. La notion de Disegno en Italie (XVe-XVI siècles). Paris : L'Harmattan.

GREENAWAY, Peter. 1996. « Annexe 2 : Les treize livres écrits sur la peau : Le Livre Premier », in The Pillow Book. Traduit par Stephen Wright, Paris : Dis voir.

GUIBERT, Hervé. 1981. « Les Lunettes à lire la pensée », in L’image fantôme. Paris : Minuit.

JEUDY, Henri-Pierre. 1998. Le Corps comme objet d'art. Paris : Armand Colin, Masson.

LAQUEUR, Thomas. 1992 [1990]. La Fabrique du sexe. Essai sur le corps et le genre en Occident. Traduit de l'anglais par Michel Gautier, Paris : Gallimard. 
NORMAN, Sally Jane. 1993. « Le "Body Art" du conceptuel au rituel ou le leurre de la présence. », in Le Corps en jeu : Spectacles, histoire, société, ASLAN, Odette (éd.). Paris : CNRS éditions.

SERRES, Michel. 2003. Les Cinq Sens. Philosophie des corps mêlés. Paris : Grasset.

SOULAGES, François. 2005 (1998). Esthétique de la photographie. La perte et le reste. Paris : Armand Colin.

THÉVOZ, Michel. 1984. Le Corps peint. Les illusions de la réalité. Genève : Skira.

WILDE, Oscar. 2000. Le Crime de Lord Arthur Savile. Étude sur le devoir, in Contes et récits. Traduit de l'anglais par Marcel Schwob, Jules Castier, Stuart Merrill, Pascal Aquien et Françis Viélé-Griffin, Paris : Le Livre de Poche.

\section{NOTES}

1. Guibert, Hervé. 1981. « Les Lunettes à lire la pensée ", in L’Image fantôme. Paris : Minuit, p. 9.

2. Serres, Michel. 2003. Les Cinq Sens. Philosophie des corps mêlés. Paris : Grasset, p. 100.

3. Je parle bien ici d'une réaction aux propos des spectateurs de mes séries antérieures. Une réaction qui peut paraître caricaturale sur les différences de genre, mais justement intéressante en ce qu'elle révèle les aprioris socio-culturels toujours en vigueur et dont je joue, que je parodie avec cette série. Sur la remise en question d'une distinction des sexes biologiques comme vérité absolue, je recommande la lecture de Laqueur, Thomas. 1992 pour la traduction. La Fabrique du sexe. Essai sur le corps et le genre en Occident. Traduit de l'anglais par Michel Gautier, Paris: Gallimard.

4. Urs Lüthi, cité par Norman, Sally Jane. 1993. «Le "Body Art" du conceptuel au rituel ou le leurre de la présence ", in Le Corps en jeu : Spectacles, histoire, société. Paris : CNRS éditions, p. 175.

5. Wilde, Oscar. 2000. Le Crime de Lord Arthur Savile - Étude sur le devoir, in Contes et récits. Traduit de l'anglais par Marcel Schwob, Jules Castier, Stuart Merrill, Pascal Aquien et Françis ViéléGriffin, Paris : Le Livre de Poche, p. 232-233.

6. Thévoz, Michel. 1984. Le Corps peint. Les illusions de la réalité. Genève : Skira, p. 8.

7. Jeudy, Henri-Pierre. 1998. Le Corps comme objet d'art. Paris : Armand Colin, p. 63.

8. Pour une analyse plus détaillée, je vous invite à lire l'article paru en juin 2016 dans la revue en ligne Plastir - Plasticités Sciences Arts: https://www.plasticites-sciences-arts.org/plastirn42-062016/

9. Soulages, François. 1998, 2005 pour la présente édition. Esthétique de la photographie. La perte et le reste. Paris : Armand Colin, p. 103.

10. Ciaravino, Joselita. 2004. Un Art paradoxal. La notion de Disegno en Italie (XVe-XVIe siècles). Paris : L'Harmattan, p. 29.

11. Ibid., p. 91.

12. Greenaway, Peter. 1996. «Annexe 2 : Les treize livres écrits sur la peau : Le Livre Premier », in The Pillow Book. Traduit par Stephen Wright, Paris : Dis voir, p. 102. 


\section{RÉSUMÉS}

Ce texte présente ma série de photographies performatives Les Transparences (2007). Cette série confronte la nature biologique des sexes à la nature performative des genres en mettant en scène des modèles Drag Queens. J'interroge alors la valeur arbitraire des associations entre sexes et genres tout en observant les lois socio-culturelles qui en dessinent les règles. Dans ma pratique artistique, j'utilise les masques, les postures stéréotypées du corps comme des discours visuels faisant de ce corps l'écran d'une mise en scène identitaire culturellement reconnaissable. J'interroge les liens entre l'art et le vivant. Mes portraits ne montrent jamais des individus, mais des corps en représentation, performatifs qui, se projetant dans une mise en scène artistique, portent leur discours sur l'identité et les façons de la revendiquer.

This text presents my series of performative photographs called Les Transparences ("Transparencies"), 2007. By staging drag queens models, this series brings face-to-face the biological nature of sex and the performative nature of genders. Thereby I question the arbitrary value of associating sex and gender while observing the sociocultural laws that regulate it. In my artistic practice, I use masks and stereotyped body postures as visual discourses that make the body into a screen on which a culturally recognisable identity is displayed. I question the relationship between art and life. My portraits never show individuals but rather performing and performative bodies that both carry discourses on identity and claim identities through an artistic staging.

\section{INDEX}

Mots-clés : photographie, performance, portrait, genre, drag queens

Keywords : photography, performance, portrait, gender, drag queens

Thèmes : Créations

\section{AUTEUR}

\section{LORRAINE ALEXANDRE}

Lorraine Alexandre est artiste plasticienne et chercheuse, docteur en art et sciences de l'art, mention arts plastiques. Spécialisée en photographie performative, elle interroge les modes de mises en scène et de réappropriations formelles du corps dans l'ensemble des arts. Elle publie en 2011 son livre Les enjeux du portrait en art. Étude des rapports modèle, portraitiste, spectateur chez L'Harmattan, collection « Champs visuels », série Théories de l'image / Images de la théorie. Elle expose en tant qu'artiste et contribue à des publications collectives régulièrement. 